\title{
ON THE SATURATION CLASS FOR SPLINE FUNCTIONS
}

\author{
FRANKLIN RICHARDS
}

\begin{abstract}
Let $\mathscr{S}_{k}\left(\Delta_{n}\right)$ be the space of piecewise polynomials of degree at most $k$ on $[0,1]$ possessing jumps at $1 / n, 2 / n, \cdots, n-1 / n$. Recently Gaier has shown that under the supremum norm $\left\|f-\mathscr{S}_{k}\left(\Delta_{n}\right)\right\|+\left\|f-\mathscr{S}_{k}\left(\Delta_{n+1}\right)\right\| \geqq c n^{-k-1}$ unless $f$ is a polynomial of degree at most $k$. Here we show if $0<\alpha \leqq 1$, then $\left\|f-\mathscr{S}_{k}\left(\Delta_{n}\right)\right\|=$ $O\left(n^{-k-\alpha}\right)$ if and oniy if $f \in C^{k}[0,1]$ and $f^{(k)}$ satisfies a Lipschitz condition of order $\alpha$. In addition, a result similar to Gaier's is given.
\end{abstract}

1. Let $\Delta: 0=x_{0}<x_{1}<\cdots<x_{n}=1$ be some subdivision of $[0,1]$. We then define the class of spline functions of degree $k$ with knots $\Delta, \mathscr{S}_{k}(\Delta)$, to be those functions $f(x)$ which reduce to an element of $\pi_{k}$ (the set of polynomials of degree at most $k$ ) on each subinterval $\left[x_{i-1}, x_{i}\right]$. In the usual definition, we also require $f \in C^{k-1}$, but that will not be assumed here.

If $f$ is a bounded real valued function on $[0,1]$, define

$$
\|f\|=\sup _{x \in[0,1]}|f(x)| \text { and }\left\|f-\mathscr{S}_{k}(\Delta)\right\|_{i}=\inf _{s \in \mathscr{S}_{k}(\Delta)}\|f-s\| .
$$

By a saturation theorem for spline functions, we mean a solution to the following problem: Let $\Delta_{n}$ be a given sequence of subdivisions of $[0,1]$. Can we then find a sequence of positive reals $\left(\alpha_{n}\right)$ and a set $\mathscr{L}_{1}^{k}$ so that

$$
\left\|f-\mathscr{S}_{k}\left(\Delta_{n}\right)\right\|=O\left(\alpha_{n}\right) \text { if and only if } f \in \mathscr{L}_{1}^{k}
$$

and

$$
\left\|f-\mathscr{S}_{k}\left(\Delta_{n}\right)\right\|=o\left(\alpha_{n}\right) \text { if and only if } f \in \pi_{k} .
$$

Gaier [1] has established (2) under the condition of uniform subdivisions, where he shows $\alpha_{n}=n^{-k-1}$. We shall solve the corresponding problem for (1) and a result of the form (2) for more general type subdivisions.

2. In this section, we assume $\Delta_{n}=\{i / n ; i=0,1, \cdots, n\}$.

THEOREM 1. Let $k$ be a given nonnegative integer, $0<\alpha \leqq 1$, and define $\mathscr{L}_{\alpha}^{k}=\left\{f ; f \in C^{k}[0,1], f^{(k)}\right.$ satisfies a Lipschitz condition of order $\left.\alpha\right\}$. Then

$$
\left\|f-\mathscr{S}_{k}\left(\Delta_{n}\right)\right\|=O\left(n^{-k-\alpha}\right) \text { if and only if } f \in \mathscr{L}_{\alpha}^{k} .
$$

Received by the editors July 6, 1971.

AMS 1970 subject classifications. Primary 41A15, 41 A40; Secondary 26A16.

Key words and phrases. Spline function, saturation class. 
The sufficiency of (3) is known (see e.g. [3]). Now suppose

$$
\left\|f-\mathscr{S}_{k}\left(\Delta_{n}\right)\right\|=O\left(n^{-k-\alpha}\right) .
$$

To show $f \in \mathscr{L}_{\alpha}^{k}$ requires a series of lemmas.

LEMMA 1. Let $S_{n} \in \mathscr{S}_{k}\left(\Delta_{n}\right)$ be a best approximation to $f$. Then there exist functions $f_{v}, f_{v}, v=0,1, \cdots, k$, such that

$$
\begin{aligned}
& S_{2^{n}}^{(\nu)}(x) \rightarrow f_{v}(x) \quad \text { uniformly for } x \notin \bigcup_{n=1}^{\infty} \Delta_{2^{n}} \\
& S_{3^{n}}^{(v)}(x) \rightarrow f_{v}(x) \quad \text { uniformly for } x \notin \bigcup_{n=1}^{\infty} \Delta_{3^{n}} .
\end{aligned}
$$

PROof. Observe that $S_{2^{n}}-S_{2^{n+1}} \in S_{k}\left(\Delta_{2^{n+1}}\right)$. Hence by using Markoff's inequality on each subinterval of $\Delta_{2^{n+1}}$, it follows that

$$
\left\|S_{2^{n}}^{(v)}-S_{2^{n+1}}^{(v)}\right\| \leqq K_{1}\left(2^{n+1}\right)^{v}\left\|S_{2^{n}}-S_{2^{n+1}}\right\| \leqq K_{2} 2^{-n(k-v+\alpha)}
$$

Therefore, if $m>n$,

$$
\left\|S_{2^{n}}^{(\nu)}-S_{2^{m}}^{(\nu)}\right\| \leqq K_{3} 2^{-n(k-v+\alpha)}
$$

and so there exist functions $f_{v}$ satisfying (5), in particular

$$
\left\|S_{2^{n}}^{(v)}-f_{v}\right\| \leqq K_{3} 2^{-n(k-v+\alpha)} .
$$

Note that $f_{v}(x)$ is defined only for $x \notin \bigcup_{n=1}^{\infty} \Delta_{2^{n}}$. The proof of (6) is nearly identical.

LEMMA 2. $f_{v}$ is a continuous function on its domain, i.e. if $x_{0} \notin \cup \Delta_{2^{n}}$, then

$$
\lim _{\varepsilon \rightarrow 0} \sup _{\left|x-x_{0}\right|<\varepsilon}\left|f_{v}(x)-f_{v}\left(x_{0}\right)\right|=0, \quad x \notin \bigcup \Delta_{2^{n}} \text {. }
$$

A similar statement holds for $\bar{f}_{v}$.

Proof. Suppose not. Then for some $x_{0}$,

$$
\lim _{\varepsilon \rightarrow 0} \sup _{\left|x-x_{0}\right|<\varepsilon}\left|f_{v}(x)-f_{v}\left(x_{0}\right)\right|=\delta>0 .
$$

But since $S_{2^{n}}^{(v)}$ is continuous on each subinterval of $\Delta_{2^{n}}$, we must have $\left\|S_{2^{n}}^{(v)}-f_{v}\right\| \geqq \delta / 2$ for all $n$, contradicting (5).

LEMMA 3. $f \in C^{k}[0,1]$.

Proof. We shall show $f^{(v)}$ exists, is continuous, and $f^{(v)}=f_{v}=\bar{f}_{v}$ (where this equality makes sense), $\nu=0,1, \cdots, k$. The proof will be by induction on $v$. 
By (4)-(6), $f(x)=f_{0}(x)$ for $x \notin \bigcup \Delta_{2^{n}}$, and $f(x)=\bar{f}_{0}(x)$ for $x \notin \bigcup \Delta_{3^{n}}$. Continuity of $f$ follows from Lemma 2 .

Now suppose $f^{(v-1)}$ is continuous. For $\xi, x \notin \bigcup \Delta_{\mathbf{x}^{i n}}$, we write

$$
\int_{\xi}^{x} f_{v}(t) d t=\int_{\xi}^{x}\left\{f_{v}(t)-S_{2^{n}}^{(v)}(t)\right\} d t+\int_{\xi}^{x} S_{2^{n}}^{(v)}(t) d t
$$

The first term on the right will $\rightarrow 0$ as $n \rightarrow \infty$. Letting $h_{i}^{v}=h_{i}^{v}(n)$ denote the jump of $S_{2^{n}}^{(v)}$ at $x_{i}=i 2^{-n}$, i.e. $h_{i}^{v}=S_{2^{n}}^{(v)}\left(x_{i}+\right)-S_{2^{n}}^{(v)}\left(x_{i}-\right)$, it follows that

$$
\int_{\xi}^{x} S_{2^{n}}^{(\nu)}(t) d t=S_{2^{n}}^{(\nu-1)}(x)-S_{2^{n}}^{(\nu-1)}(\xi)-\sum^{\prime} h_{i}^{\nu-1}
$$

where $\Sigma^{\prime}$ means we sum over those $i$ such that $i 2^{-n}$ lies between $\xi$ and $x$. By (7) and since $f^{(v-1)}=f_{v-1}$ is continuous, one has

$$
\left|h_{i}^{\nu-1}\right| \leqq 2 K_{3^{2}} 2^{-n(k-v+1+\alpha)}, \quad i=1,2, \cdots, 2^{n}-1
$$

Hence

$$
\sum^{\prime}\left|h_{i}^{v-1}\right| \leqq 2 K_{3} 2^{-n(k-v+\alpha)} \leqq 2 K_{3} 2^{-n \alpha}
$$

Letting $n \rightarrow \infty$ in (8), we have from (5), (9), and (10),

$$
\int_{\xi}^{x} f_{v}(t) d t=f_{v-1}(x)-f_{v-1}(\xi) .
$$

Lemma 2 and the induction hypothesis now impiy $f^{(v)}(x)=f_{v}(x)$. Similarly $f^{(v)}(x)=\bar{f}_{v}(x)$ for $x \notin \bigcup \Delta_{3^{n}}$, and so $f^{(v)}$ is continuous.

LEMMA 4. $f^{(\dot{i})}$ satisfies a Lipschitz condition of order $\alpha$.

Proof. Suppose $x, y \notin \cup \Delta_{2^{n}}, x<y$. Then there exists $N=N(x, y)$ such that at least one and at most two subintervals of $\Delta_{2^{N}}$ lie strictly between $x$ and $y$. Let us choose points $Z_{0}, Z_{1}, \cdots, Z_{2 r+1}, r=2$ or 3 , satisfying the following conditions:

$$
\begin{aligned}
r=2: \frac{q}{2^{N}} & <x<Z_{1}<\frac{q+1}{2^{N}}<Z_{2}<Z_{3}<\frac{q+2}{2^{N}} \\
& <Z_{4}<y<\frac{q+3}{2^{N}}, \\
r=3: \frac{q}{2^{N}} & <x<Z_{1}<\frac{q+1}{2^{N}}<Z_{2}<Z_{3}<\frac{q+2}{2^{N}} \\
& <Z_{4}<Z_{5}<\frac{q+3}{2^{N}}<Z_{6}<y<\frac{q+4}{2^{N}},
\end{aligned}
$$




$$
\begin{gathered}
Z_{2 i}-Z_{2 i-1}<\min \left\{\frac{1}{2^{N} 3^{N}}, \frac{q+1}{2^{N}}-x, y-\frac{q+r}{2^{N}}\right\}, \quad i=1, \cdots, r, \\
Z_{i} \notin\left(\cup \Delta_{2^{n}}\right) \cup\left(\cup \Delta_{3^{n}}\right), \\
Z_{0}=x, \quad Z_{2 r+1}=y .
\end{gathered}
$$

Now

$\left|f^{(k)}(y)-f^{(k)}(x)\right| \leqq \sum_{i=0}^{r}\left|f^{(k)}\left(Z_{2 i+1}\right)-f^{(k)}\left(Z_{2 i}\right)\right|+\sum_{i=1}^{r}\left|f^{(k)}\left(Z_{2 i}\right)-f^{(k)}\left(Z_{2 i-1}\right)\right|$.

Since $Z_{2 i}$ and $Z_{2 i+1}$ lie on the same subinterval of $\Delta_{2^{N}}$, and $S_{2 N}^{(k)}$ is a step function, we have, by (7),

$$
\begin{gathered}
\left|f^{(k)}\left(Z_{2 i+1}\right)-f^{(k)}\left(Z_{2 i}\right)\right| \leqq \\
+\left|f_{k}\left(Z_{2 i+1}\right)-S_{2^{\nu}}^{(k)}\left(Z_{2 i+1}\right)\right|+\left|S_{2^{N}}^{(k)}\left(Z_{2 i+1}\right)-S_{2^{N}}^{(k)}\left(Z_{2 i}\right)\right| \\
+\mid S_{2^{N}}^{(k)}\left(Z_{2 i}\right)-f_{k}\left(Z_{2 i}\right) ! \leqq 2 K_{3} 2^{-N \alpha} .
\end{gathered}
$$

By (12), $Z_{2 i}$ and $Z_{2 i-1}$ lie on the same subinterval of $\Delta_{3^{\mathrm{N}}}$. Hence

and so

$$
\left|f^{(k)}\left(Z_{2 i}\right)-f^{(k)}\left(Z_{2 i-1}\right)\right| \leqq 2 K_{4} 3^{-N \alpha}
$$

$$
\left|f^{(k)}(y)-f^{(k)}(x)\right| \leqq 8 K_{3} 2^{-N \alpha}+6 K_{4} 3^{-N \alpha} .
$$

But $2^{-v}<y-x$. Therefore

$$
\left|f^{(k)}(y)-f^{(k)}(x)\right| \leqq\left(8 K_{3}+6 K_{4}\right)|y-x|^{\alpha} .
$$

This completes the proof of the lemma and the theorem.

ReMARK. The assumption that our splines be elements of $C^{k-1}$ considerably simplifies the proof.

3. It is not too difficult to see that the preceding proof may be altered slightly to establish (2). However the following result of Gaier is considerably stronger.

THEOREM 2 (GAIER). Suppose $f \neq \pi_{k}$. Then there exists a constant $c=c(f)>0$ such that

$$
\left\|f-\mathscr{S}_{k}\left(\Delta_{n}\right)\right\|+\left\|f-\mathscr{S}_{k}\left(\Delta_{n+1}\right)\right\| \geqq c / n^{k+1} .
$$

Before considering a version of Theorem 2 for more arbitrary subdivisions, an example seems in order. Let

$$
\begin{aligned}
F(x)=\left(x-\frac{1}{2}\right)_{+}^{k} & =\left(x-\frac{1}{2}\right)^{k}, & & x \geqq \frac{1}{2} \\
& =0, & & x<\frac{1}{2} .
\end{aligned}
$$

Since $F \in \mathscr{S}_{k}\left(\Delta_{2 n}\right)$, we see that (15) cannot be improved to $\left\|f-\mathscr{S}_{k}\left(\Delta_{n}\right)\right\| \geqq$ $c / n^{k+1}$. This is due to the fact that $F$ is not sufficiently smooth, as will be demonstrated in Theorem 3. 
We now introduce some notation. The subdivisions $\Delta_{n}$ will be of the form $\Delta_{n}: 0=x_{0}^{n}<x_{1}^{n}<\cdots<x_{n}^{n}=1$. Let $\left\|\Delta_{n}\right\|=\max _{i}\left(x_{i}^{n}-x_{i-1}^{n}\right)$ and $\left\langle\Delta_{n}\right\rangle=\min _{i}\left(x_{i}^{n}-x_{i-1}^{n}\right)$. Then we may state

THEOREM 3. Let $\Delta_{n}$ be a sequence of subdivisions satisfying

$$
\left\|\Delta_{n}\right\| /\left\langle\Delta_{n}\right\rangle \leqq \beta<\infty, \quad n=1,2, \cdots .
$$

Then if $f \in C^{k+1}[0,1]$ and $f \notin \pi_{k}$, there exists a constant $c=c(f)>0$ such that

$$
\left\|f-\mathscr{S}_{k}\left(\Delta_{n}\right)\right\| \geqq c / n^{k+1}
$$

Proof. Suppose (17) does not hold. Then there exist a sequence of subdivisions $\Delta_{r_{i}}$ and a function $\phi(n)$ satisfying

$$
\left\|f-\mathscr{S}_{k}\left(\Delta_{n_{i}}\right)\right\| \leqq \phi\left(n_{i}\right) n_{i}^{-k-1}
$$

and $\phi(n) \rightarrow 0$ as $n \rightarrow \infty$.

On each subinterval $\left[x_{j}^{n_{i}}, x_{j+1}^{n_{i}}\right]$ of $\Delta_{n_{i}}$, choose $k+2$ equally spaced points $y_{0}, y_{1}, \cdots, y_{k+1}$ such that $y_{k+1}-y_{0}=\left\langle\Delta_{n_{i}}\right\rangle$. Then using the Steffenson notation for divided differences, one finds

$$
\begin{aligned}
& S_{n_{i}}\left(y_{0}, \cdots, y_{k+1}\right)-f\left(y_{0}, \cdots, y_{k+1}\right) \\
& \quad=\frac{1}{(k+1) !} \frac{(k+1)^{k+1}}{\left\langle\Delta_{n_{i}}\right\rangle^{k+1}} \sum_{v=0}^{k+1}(-1)^{v}\left(\begin{array}{c}
k+1 \\
v
\end{array}\right)\left\{S_{n_{i}}\left(y_{v}\right)-f\left(y_{v}\right)\right\}
\end{aligned}
$$

where $S_{n_{i}} \in \mathscr{S}_{k}\left(\Delta_{n_{i}}\right)$ is a best approximation to $f$. But since $S_{n_{i}} \in \pi_{k}$ on $\left[x_{j}^{n_{i}}, x_{j+1}^{n_{i}}\right], f \in C^{k+1}[0,1]$, and using (16) and (18) we obtain

$$
\begin{aligned}
\left|f^{(k+1)}\left(\xi_{j}^{n_{i}}\right)\right| & \leqq[2(k+1)]^{k+1} \frac{n_{i}^{-k-1}}{\left\langle\Delta_{n_{i}}\right\rangle^{k+1}} \phi\left(n_{i}\right) \\
& \leqq\{2 \beta(k+1)\}^{k+1} \phi\left(n_{i}\right)
\end{aligned}
$$

where $x_{j}^{n_{i}}<\xi_{j}^{n_{i}}<x_{j+1}^{n_{i}}$. Since $\left\|\Delta_{n_{i}}\right\| \rightarrow 0$, every point of $[0,1]$ is a limit point of some infinite subset of $\left\{\xi_{j}^{n_{i}}, j=0,1, \cdots, n_{i}-1 ; i=1,2, \cdots\right\}$. Hence if $f \in C^{k+1}[0,1]$, by $(18)$ and $(20)$ we have $f^{(k+1)}(x) \equiv 0, x \in[0,1]$, i.e. $f \in \pi_{k}$.

ACKnowledgement. I am indebted to Professor Z. Ditzian for several valuable suggestions.

\section{REFERENCES}

1. D. Gaier, Saturation bei Spline-Approximation und Quadratur, Numer. Math. 16 (1970), 129-140. 
2. V. A. Popov and Bl. Kh. Sendov, Classes characterized by best-possible approximation by spline functions, Math. Notes 8 (1970), no. 2, 550-557 (translated from Mat. Zametki).

3. F. Richards, Convergence of natural spline interpolants on uniform subdivisions (to appear).

Department of Mathematics, University of Alberta, Edmonton, Alberta, CANADA 